\title{
A Functional Model for the SONATA Switchless Optical Network
}

\author{
S. De Maesschalck, P. Demeester \\ Dep. of Information Technology, University of Gent - IMEC \\ Sint-Pietersnieuwstraat 41, B-9000 Gent, Belgium \\ Tel: +3292673590, Fax: +3292673599 \\ E-mail: sophie.demaesschalck@intec.rug.ac.be
}

Key words: Switchless optical network, functional model, management advantages

\begin{abstract}
In this paper we clarify the concept of the switchless optical network developed in the framework of the ACTS project SONATA. The functional model we developed for this kind of network is explained. Some management advantages of this kind of network structure are discussed.
\end{abstract}

\section{INTRODUCTION}

The growing need for bandwidth has led to the introduction of WDM (Wavelength Division Multiplexing) in the core network. This technique is for the moment mainly used in point-to-point links but in the future the introduction of optical cross-connects and add/drop multiplexers, replacing the current electrical nodes, may be expected. The general structure of the network however will not change: there will still be a distinction between access and core part of the network and between transport and switching function. The core network will consist of multiple large and complex optical nodes connected by high-capacity optical links in a mesh or ring structure. Several switching technologies (e.g. ATM, IP,...) will work in parallel on this transport layer. The access network will be based on different solutions like ATM PONs, xDSL on copper pairs,.... 
The switchless optical network proposed here, is a new and simplified network structure developed in the framework of the ACTS project SONATA to avoid the need for large and fast switching nodes in the network [1], [2]. The distinction between access and transport part of the network has completely disappeared. It is basically a single layer all-optical network providing end-to-end optical connections between a large number of terminals distributed over a wide geographical area. This multiple-access network employs both time and wavelength agility at the terminals (a TDMA/WDMA protocol) to permit customers to send both connectionoriented and datagram traffic over the network.

The network has a very simple structure with all traffic passing through a single node providing passive wavelength routing and, optionally, actively controlled wavelength conversion. The network is switchless in the sense that there is no need for electrical or optical switches or cross-connects in the network, except for the single central routing node.

The aim of this paper is to provide a functional model for this type of network based on the modelling methodology described in ITU-T Recommendation G.805 [3]. The use of this type of network modelling is very powerful as a foundation for developing network management specifications [4]. In section 2 the structure and the concept of SONATA are further explained. In section 3 the layer architecture is discussed. Section 4 describes the functional model developed for this network. In section 5 some management advantages of the switchless optical network structure are discussed.

\section{STRUCTURE AND CONCEPT}

The structure of a basic switchless optical network is shown in Figure 1. On the figure the network structure has been unfolded in a downstream and an upstream part to enhance its legibility. The direction of all signals is from left to right.

The heart of the switchless optical network is the passive wavelength routing node (PWRN) to which a number $\left(\mathrm{N}_{\mathrm{d}}\right)$ of PONs (Passive Optical Network) are connected. Each PON has a separate fibre for the upstream and downstream direction. The customer's terminals, each containing a wavelength agile sender and transmitter, are attached to the branches of those PONs. Each PON can transfer data to each other PON (including his own) on a dedicated wavelength that traverses the central node. As the terminals of each PON have to be able to communicate with the terminals of all PONs, a terminal needs a tuneable sender/receiver pair able to transmit/receive on $\mathrm{N}_{d}$ different wavelengths. On top of this, each 
wavelength between a PON pair is shared by the various terminals of that PON which results in the need for a TDMA protocol. Time is divided into fixed-length timeslots and in a single timeslot the terminals of a PON need to use a different wavelength. A sequence of timeslots is further structured into fixed-size time frames on each wavelength channel.

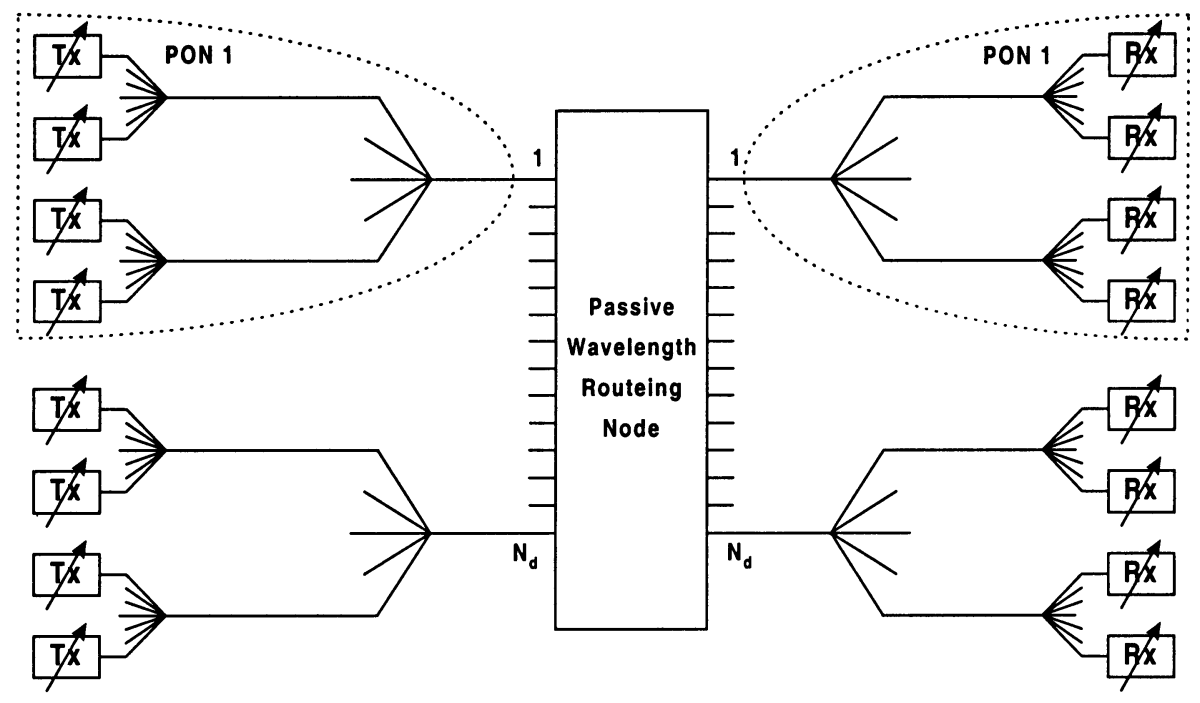

Figure 1. Basic structure of the switchless optical network

The different optical channels in a PON are multiplexed by the successive combiners into a single WDM signal per PON. The network thus uses a double multiplexing strategy: one in the time domain and one in the wavelength domain. This WDM signal enters the router at an in-going port, is demultiplexed and the optical channels are routed to the correct outgoing port, based solely on their wavelength, where they are multiplexed with the channels routed to the same outgoing port. This routing principle is illustrated in Figure 2 for the switchless network structure of Figure 1. The WDM signal leaving the central node at each outgoing port is broadcast to all terminals of the PON to which the destination terminal belongs. 


\begin{tabular}{|c|c|c|c|c|c|}
\hline & \multicolumn{4}{|c|}{$N_{d}$ outgoing ports } \\
\hline & & $1^{\prime}$ & $2^{\prime}$ & $N_{d-1}{ }^{\prime}$ & $N_{d}^{\prime}$ \\
\hline \multirow{3}{*}{ 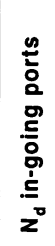 } & 1 & $\lambda_{1}$ & $\lambda_{2}$ & $\lambda_{\mathrm{Nd}-1}$ & $\lambda_{\mathrm{Nd}}$ \\
\hline & 2 & $\lambda_{\text {No }}$ & $\lambda_{1}$ & $\lambda_{\text {Nd-2 }}$ & $\lambda_{\text {Nd-1 }}$ \\
\hline & $N_{d-1}{ }^{\prime}$ & $\lambda_{3}$ & $\lambda_{4}$ & $\lambda_{1}$ & $\lambda_{2}$ \\
\hline & $N_{d}$ & $\lambda_{2}$ & $\lambda_{3}$ & $\lambda_{\mathrm{Nd}}$ & $\lambda_{1}$ \\
\hline
\end{tabular}

Figure 2. Fixed routing of the wavelength channels in the central node for the basic structure

As illustrated in Figure 3 the SONATA switchless optical network has a number of additional features, described below.

In general cascaded optical amplifiers are required in the branches of the PONs due to the large geographical area covered and the large splitting ratio. In such large split "hyper-PONs" a noise gating functional block has to be placed after those amplifiers and before the last combiner in the upstream direction to avoid accumulation of ASE noise from the not-used branches during each timeslot [5].

The central node also needs a connection to the central network controller, which is responsible for the allocations of the time slots in such a way that the network is collision free.

In order to increase the flexibility and the available bandwidth between a PON pair, additional wavelength channels $\left(\mathrm{N}_{\mathrm{i}}\right)$ are introduced that are dynamically allocated to a PON pair depending on the traffic requirements. The multiplex coming from a PON now contains $\mathrm{N}_{d}+\mathrm{N}_{\mathrm{i}}$ optical channels. The central node therefore needs $\mathrm{N}_{\mathrm{i}}$ extra in-going and outgoing ports, called dummy ports, each of which is connected to an array of wavelength converters. The complete structure of the SONATA switchless optical network is shown in Figure 3. 


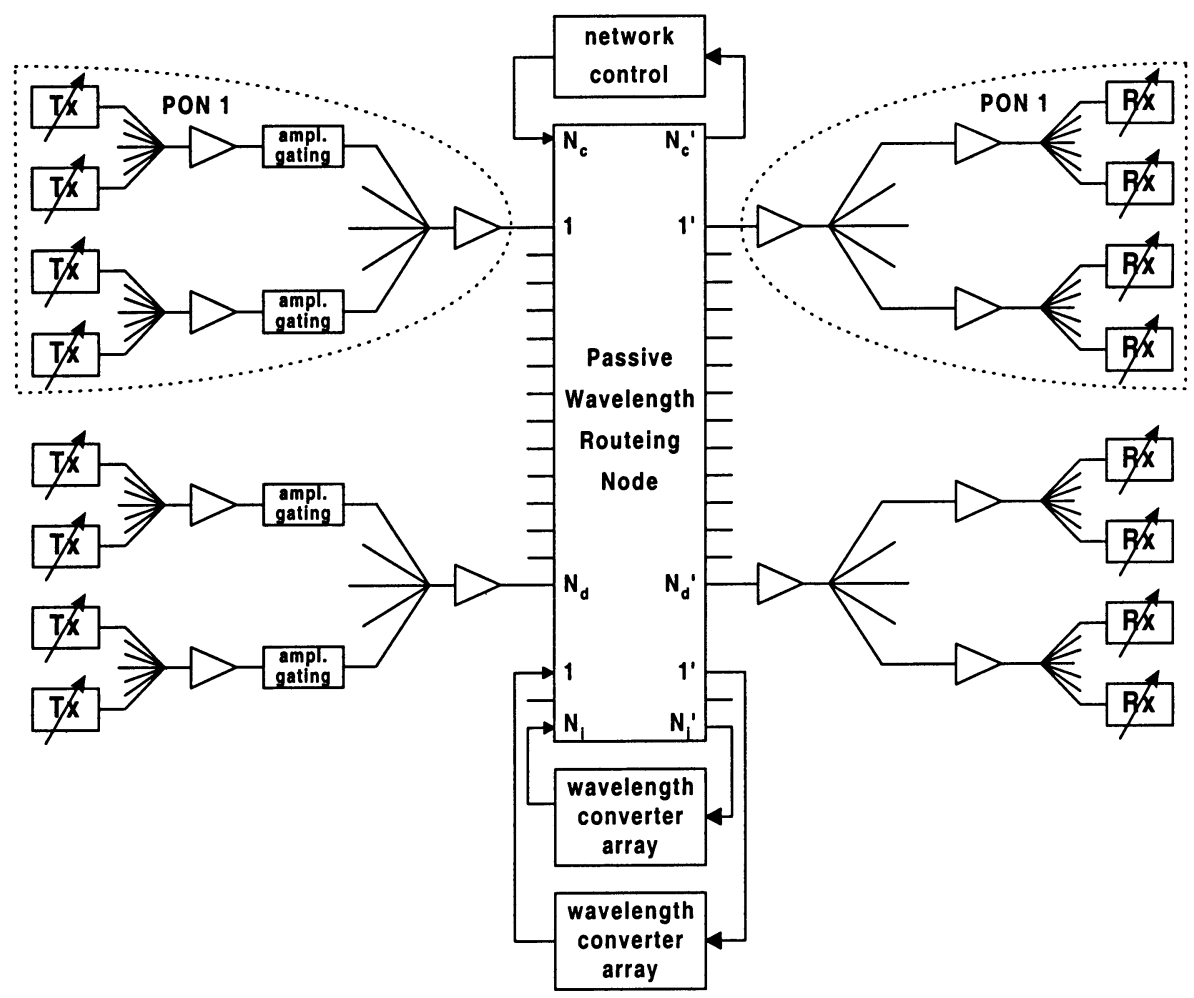

Figure 3. Complete structure of the switchless optical network

The extra optical channels are routed across the PWRN to the correct outgoing dummy port, based on their wavelength and pass through the actively controlled wavelength converter block. Here their wavelength is translated to the wavelength needed to reach the correct outgoing port of the PWRN. The routing table of Figure 2 is extended in Figure 4 for the complete structure.

To differentiate them from the (direct) wavelength channels that only pass the PWRN once, these wavelength channels are called indirect wavelength channels, because they also pass trough the wavelength converter bank. 


\begin{tabular}{|c|c|c|c|c|c|c|}
\hline & \multicolumn{4}{|c|}{$\mathrm{N}_{\mathrm{d}}$ outgoing ports } & \multicolumn{2}{|c|}{$\begin{array}{l}N_{1} \text { outgoing dummy } \\
\text { ports }\end{array}$} \\
\hline & $1^{\prime}$ & $2^{\prime}$ & $N_{d-1}{ }^{\prime}$ & $N_{d}{ }^{\prime}$ & $1^{\prime}$ & $N_{i}^{\prime}$ \\
\hline 1 & $\lambda_{1}$ & $\lambda_{2}$ & $\lambda_{\mathrm{Nd}-1}$ & $\lambda_{\mathrm{Nd}}$ & $\lambda_{\mathrm{Nd}+1}$ & $\lambda_{\mathrm{Nd}+\mathrm{Ni}}$ \\
\hline$\stackrel{\circ}{0}$ & $\lambda_{\mathrm{Nd}+\mathrm{Ni}}$ & $\lambda_{1}$ & $\lambda_{\mathrm{Nd}-2}$ & $\lambda_{\mathrm{Nd}-1}$ & $\lambda_{\mathrm{Nd}}$ & $\lambda_{\mathrm{Nd}+\mathrm{Ni}}$ \\
\hline \multirow[t]{2}{*}{$z^{0}$} & $\lambda_{\mathrm{Ni}+3}$ & $\lambda_{\mathrm{Ni}+4}$ & $\lambda_{1}$ & $\lambda_{2}$ & $\lambda_{3}$ & $\lambda_{\mathrm{Ni}+2}$ \\
\hline & $\lambda_{N+2}$ & $\lambda_{\mathrm{N}+3}$ & $\lambda_{\mathrm{Nd}+\mathrm{Ni}}$ & $\lambda_{1}$ & $\lambda_{2}$ & $\lambda_{\mathrm{Ni}+1}$ \\
\hline \multirow{2}{*}{ 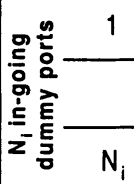 } & $\lambda_{\mathrm{N}+1}$ & $\lambda_{\mathrm{Ni}+2}$ & $\lambda_{\mathrm{Nd}+\mathrm{Ni}-1}$ & $\lambda_{\mathrm{Nd}+\mathrm{Ni}}$ & $\lambda_{1}$ & $\lambda_{\mathrm{N}}$ \\
\hline & $\lambda_{2}$ & $\lambda_{3}$ & $\lambda_{\text {Nd }}$ & $\lambda_{\mathrm{Nd}+1}$ & $\lambda_{\mathrm{Nd}+2}$ & $\lambda_{1}$ \\
\hline
\end{tabular}

Figure 4. Fixed routing of the wavelength channels in the central node for the complete structure

With one additional wavelength channel $(\mathrm{Ni}=1)$ it is e.g. possible to double the available bandwidth for in-PON traffic. We will explain with an example how this works. Traffic within PON 1 is transmitted on the direct wavelength $\lambda_{1}$. The PWRN routes this optical channel from port 1 to port 1 '.With one additional wavelength available data from PON 1 is sent on $\lambda_{\mathrm{Nd}+1}$. It enters the PWRN on port 1, like all traffic coming from PON1, and is routed to outgoing dummy port 1' (see figure 4). In order to be transmitted to PON1, it has to reach outgoing port 1'. Therefore it has to re-enter the PWRN with wavelength $\lambda_{\mathrm{Ni}+1}=\lambda_{2}$. Thus, the wavelength converter has to translate $\lambda_{\mathrm{Nd}+1}$ to $\lambda_{2}$.

To further increase the flexibility and not to allocate an entire wavelength when only a fraction of its bandwidth is needed, the indirect wavelengths will be shared among different PON pairs. In this way one extra optical channel can e.g. provide extra capacity between PONa and PONb in the first half of the frame and between PONa and PONc in the second half.

A national scale switchless optical network could have following dimensions:

- $\mathrm{N}_{\mathrm{d}}=400$ ports of the central node are connected to a PON

- Each PON is connected to 50000 terminals

- $\mathrm{N}_{\mathrm{c}}=1$ port of the PWRN is connected to the network controller

- $\mathrm{N}_{\mathrm{i}}=400$ ports of the central node are connected to wavelength converter blocks 
- The central node is a $801 \times 801$ routing node with 801 wavelength channels on each in-going and outgoing port and $0,5 \mathrm{~nm}$ spacing

- The maximum distance between two terminals is about $1000 \mathrm{~km}$.

- Burst mode operation at $622 \mathrm{Mbit} / \mathrm{s}$ on an optical channel.

- $10 \mathrm{Mbit} / \mathrm{s}$ total average rate per terminal

- $622 \mathrm{Mbit} / \mathrm{s}$ maximum rate per terminal

- 200Tbit/s maximum network throughput

\section{LAYER ARCHITECTURE}

A first important task is to find a suitable layering architecture for the switchless optical network. As mentioned before this network is considered a single-layer network, so it seems contradictory to talk about layering. Perhaps the term single-technology network would be better suited than single-layer network as the data transferred over the network stays in optical form from source to destination terminal. But within this optical transmission format several layers can be distinguished with a distinct functionality.

The SONATA concept is based on a mixture of WDM and TDM techniques to allow interconnection between the terminals of the various PONs. It seems a logical choice to provide separate layer networks for both the wavelength and the time dimension. The layering architecture for the switchless network consists then of the Optical Transport Network layers [6] (OTS, OMS and OCh layers) with a TDM layer on top of them.

\section{FUNCTIONAL MODEL}

Based on the layering architecture we developed a functional model for the components of a switchless optical network. Figure 5 shows this functional model for the network of Figure 3. The model will be explained for the different elements encountered in the network.

\section{Terminal transmitter}

At the terminal transmitter, the client data format is adapted to the timeslot format used in the switchless optical network and stored in the terminal buffer. Based on the allocation scheme received from the central network controller, the wavelength agile transmitter sends each timeslot of information in a particular timeslot of the frame on a particular wavelength. The task of the transmitter is thus threefold and spread out over all layers: adaptation of the data to timeslots at the TDM/client adaptation function, 
selection of the right timeslot in the OCh/TDM adaptation function, selection of the correct wavelength on which to transmit at the OMS/OCh adaptation function.

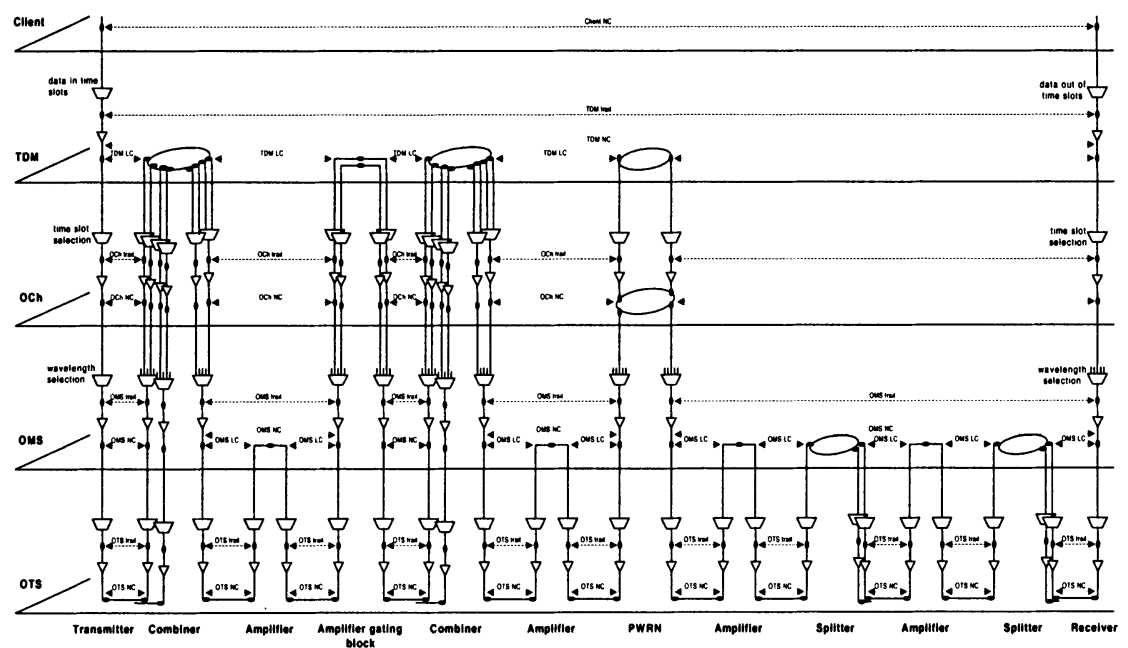

Figure 5. Functional model for the switchless optical network

\section{Combiner}

At the combiner, the signals coming from several terminals within a PON are combined. This component acts as a TDM switch whereby the timeslots on the same wavelength but in a different optical multiplex section are routed to a single transit group that is associated with a single adaptation function which time multiplexes the timeslots into the outgoing optical channel [4]. Those optical channels are then multiplexed together in on optical multiplex. This is illustrated in Figure 6.

\section{Amplifier}

The next network element encountered by the signal transmitted is the optical amplifier. Since the WDM signal is amplified as a whole, the functionality of this component has to be represented at the OMS level.

\section{Amplifier gating block}

At the amplifier gating block, the optical signal is demultiplexed. This amplifier gating block is responsible for gating the optical burst signal so that the transmission path is interrupted when no signal is present. Since gating is done timeslot by timeslot, the signal needs to be adapted out of the lower layers into the TDM layer. 


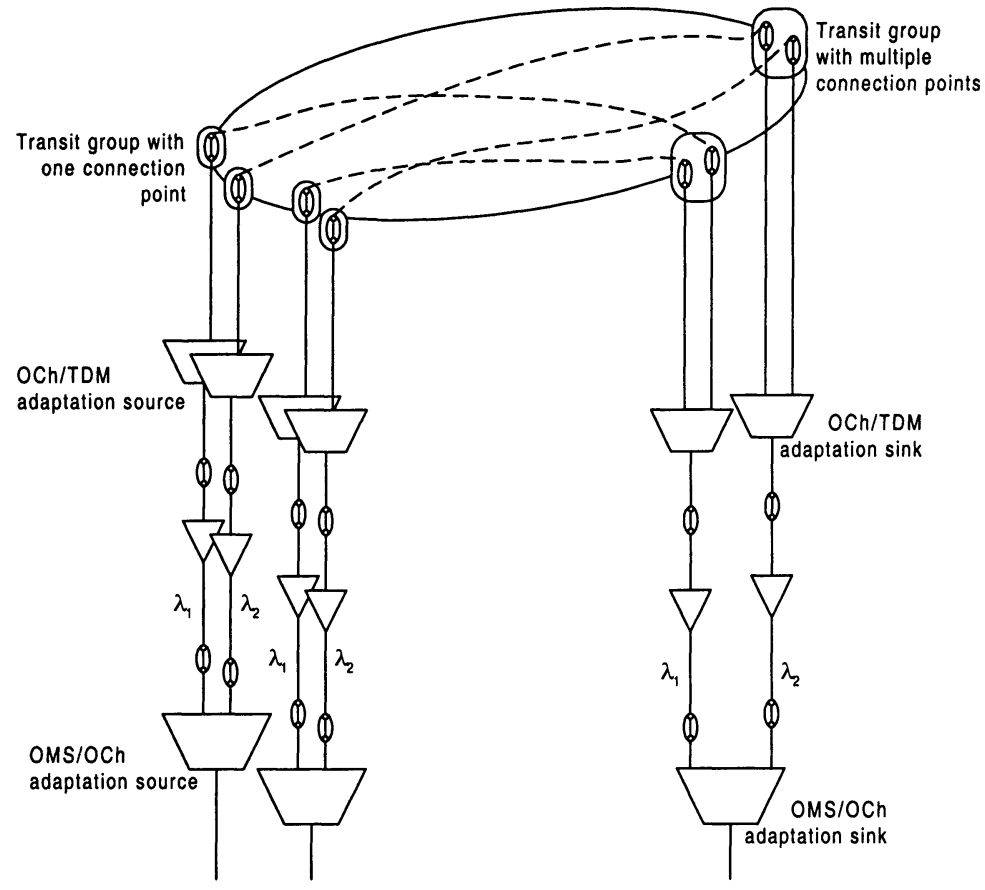

Figure 6. A combiner

\section{PWRN}

The signal then passes the central passive wavelength routing node. A mentioned before, a timeslot can be transmitted on a direct or on an indirect wavelength. In the former case the timeslot passes trough the central node only once and its wavelength cannot be changed. In the latter case the timeslot runs trough a wavelength converter. Besides this, an indirect wavelength channel can be dynamically reconfigured. This means that during one timeslot the wavelength interconnects PONx and PONy, while one timeslot later it provides interconnection between PONx and PONz. This difference has as consequence that on a direct wavelength routing is solely based on the wavelength, which has to be represented at the OCh level, while on an indirect wavelength routing is performed on a timeslot basis, which has to be represented at the TDM level.

The functional model of the PWRN is illustrated in Figure 7 for a switchless network with two PONs and one additional wavelength. 


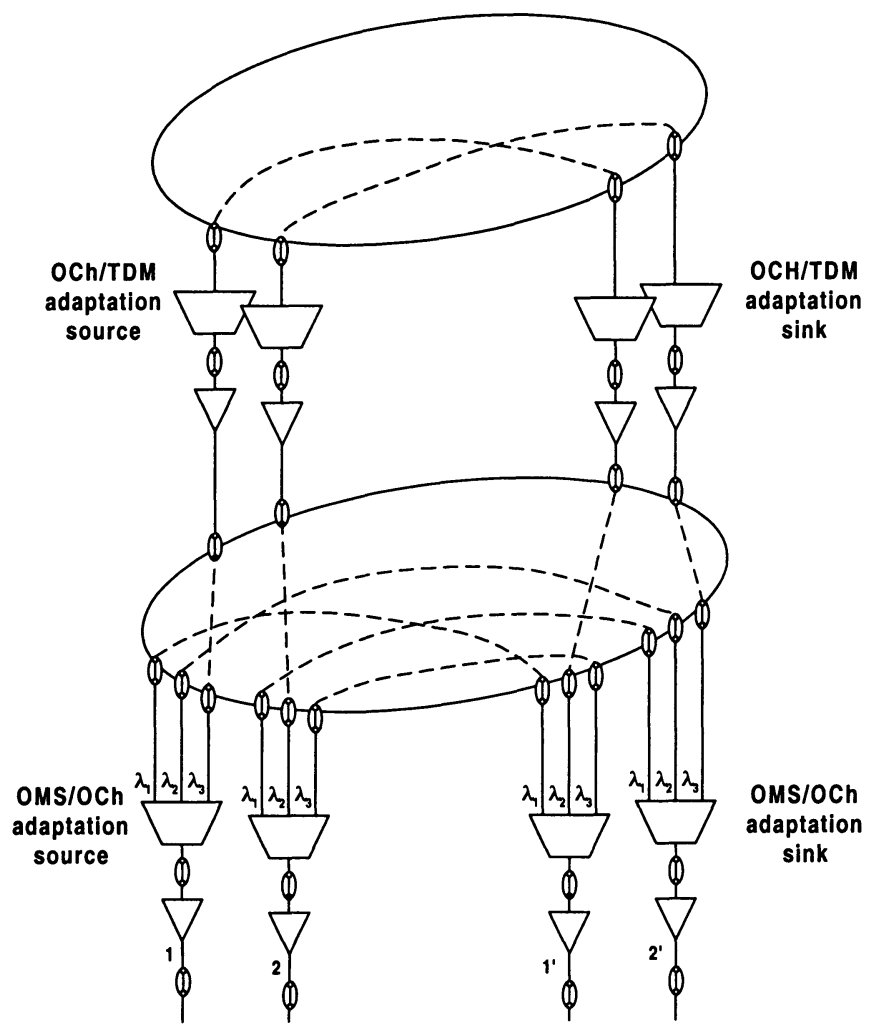

Figure 7. The PWRN

We take a look at what happens with the traffic coming from PON1. The multiplex section at in-going port 1 is demultiplexed into three optical channels. $\lambda_{1}$ is routed directly to outgoing port $1^{\prime}$ and $\lambda_{2}$ is routed directly to outgoing port 2' at the $\mathrm{OCH}$ layer. The routing in the matrix at this layer is fixed. $\lambda_{3}$ is the indirect wavelength. Some timeslots per frame on this wavelength must be routed to outgoing port 1', some to outgoing port 2'. Therefore the optical channel has to be adapted to the TDM layer, where the individual timeslots are routed correctly. The routing in the matrix at the TDM layer is not fixed but will change over time.

\section{Splitter}

In the downstream direction the first new network element is the splitter. The signal at the entrance of the splitter is a multiplex of $\mathrm{N}=\mathrm{N}_{\mathrm{d}}+\mathrm{N}_{\mathrm{i}}$ wavelengths. The traffic entity that is broadcast to each of the branches of the splitter is the characteristic information of the optical multiplex section. 
The splitter therefore provides a broadcast function at this level via an OMS subnetwork [4].

\section{Terminal receiver}

Finally the time slotted signal reaches the destination terminal. At the receiver, the right wavelength is chosen from the optical multiplex (based again on an allocation scheme coming from the central network controller) at the OMS/OCh adaptation function. The right timeslot in the frame is selected at the OCh/TDM adaptation function. The information in the timeslot is finally adapted out of the timeslot format back into the client layer transmission format.

\section{MANAGEMENT ADVANTAGES}

The switchless optical network is basically a single-technology all-optical network. Because of this, it has some management advantages not found in other network structures.

The shared access concept usually found in access networks is here extended to the entire network. All resources of the network are shared which results in a lower cost compared to more traditional networks.

Data, in the format of fixed length timeslots, is transported over the network from transmitting to receiving terminal without buffering. The timeslots to be transmitted are buffered at the edge of the network and once a timeslot is taken out of this buffer it is transmitted without any further form of buffering across the entire network until it reaches the terminal buffer at the other end of the network.

The timeslot format used can transport all kinds of formats and protocols, both connection-oriented and connectionless. In the case of connectionoriented traffic, an integer number of slots per frame is requested at connection set-up. At termination of the connection a deallocation request has to be sent to release these time-slots. For datagram traffic, a number of slots is allocated for the duration of one frame only.

Because of the reduced number of architecture layers in the network, there are no problems due to interworking and co-ordination of the management system of different technologies.

\section{CONCLUSION}

The switchless optical network represents an alternative approach to networking meant to drastically simplify the structure of future broadband 
networks. It is a single layer network in which all switching functionality is removed from the network nodes and distributed at the terminals, ending in a network structure with a single node.

The paper presented, for the first time, a functional model for this network in a four-layer architecture. The model for the various network components was discussed. Some management advantages of this network structure were discussed.

\section{ACKNOWLEDGEMENTS}

This work has been supported by the European Union ACTS Program through the project SONATA. The authors warmly acknowledge all their colleagues in the SONATA project.

\section{REFERENCES}

[1] A.M. Hill, S. Carter, J. Armitage, M.Shabeer, R.A. Harmon and P. Roise, "A scalable and switchless optical network structure employing a single $32 \times 32$ free-space grating multiplexer", IEEE Photonics Technology Letters, Vol.8, No.4, P 596-572, 1996

[2] N.P. Caponio, A. M. Hill, R. Sabella, "Switchless optical network for advanced transport architecture, IEEE/LEOS'98, Orlando, FL, USA, December 1998

[3] ITU-T Rec. G.805, "Generic Functional Architecture of Transport Networks”, November 1995

[4] Private communication with A. McGuire, BT, September 1999

[5] A.M. Hill, "WDM networking in the access network and beyond", ECOC'95 Optical Networking Forum Workshop, Brussels, $22^{\text {nd }}$ September 1995, paper S1.4

[6] ITU-T Draft Rec. G.872, “Architecture of Optical Transport Networks”, February 1999 